\title{
Young modulus dependence of nanoscopic friction coefficient in hard coatings
}

\author{
Elisa Riedo a) and Harald Brune \\ Institut de Physique des Nanostructures, Ecole Polytechnique Fédérale de Lausanne, \\ CH-1015 Lausanne, Switzerland
}

(Received 28 April 2003; accepted 15 July 2003)

\begin{abstract}
We present an atomic force microscope study of nanoscopic sliding friction on diamond, diamond-like carbon, and on three $\mathrm{CrN}$ thin films with varying hardness obtained by different growth temperatures. For the $\mathrm{CrN}$ films, we show that the changes in the friction coefficient can be traced back to variations of the Young modulus. More generally, we show for all samples investigated and in wearless regime, that the nanoscopic friction coefficient is directly linked to the Young modulus. (C) 2003 American Institute of Physics. [DOI: 10.1063/1.1609234]
\end{abstract}

A fundamental understanding of friction at micro- and down to the nanoscale is crucial for the reliability of technological devices with miniature moving parts, such as microelectromechanical systems and hard-disk drives. ${ }^{1,2}$ A better understanding of the relationship between friction at the nanoscale and material mechanical properties is expected to enable improvements in the design and operation of micromechanical devices. Common experience and scientific studies show that mechanical properties strongly affect the tribological performance of solid surfaces. ${ }^{3,4}$ However, a conclusive understanding of the correlation between mechanical and tribological properties is still lacking. The main problem in former macroscopic friction measurements was that wear and plastic deformations rendered insight in the intrinsic interfacial phenomena difficult. ${ }^{2}$ The atomic force microscope (AFM) has shown to be a very useful technique to study friction forces in the wearless regime and at the nanoscopic scale. ${ }^{1}$ Nevertheless, only very few AFM studies have investigated hardness or elasticity dependence of friction forces. ${ }^{5,6}$

Friction forces are proportional to the contact area, thus, it is intuitive that a more compliant material, into which the AFM tip is likely to penetrate deeper, yields a higher friction force. Following the theory of Bowden and Tabor, ${ }^{7}$ the friction force $F_{F}$ can be expressed as

$$
F_{F}=\tau A,
$$

where $A$ is the contact area and $\tau$ the critical shear strength defined as the shear force per unit area (or per atom) required to shear the interface, i.e., to cause sliding in a stick and slip motion. ${ }^{1}$ For small loads $\tau$ is to a good approximation independent of the load. A can be computed, for the case of a spherical tip in contact with a flat surface, using contact mechanical continuum models. ${ }^{8}$ In these models the contact area depends on the applied normal load $F_{N}$, the tip radius $R$, the Young moduli $E$, and Poisson's ratios $\nu$ of the tip and surface. Unfortunately, during a friction force experiment contributions from the contact area and from the shear strength, are convoluted. ${ }^{9,10}$ This is one of the reasons why

${ }^{a)}$ Electronic mail: elisa.riedo@physics.gatech.edu even in wearless and single asperity contact experiments it is not trivial to relate friction forces to the Young modulus of the material investigated. ${ }^{5}$

In this letter, we study sliding friction forces between a Si AFM tip and diamond, diamond-like carbon (DLC), and $\mathrm{CrN}$ thin films, i.e., materials used in industry as hard coatings. ${ }^{2,11}$ We demonstrate that the variations of the friction coefficient in $\mathrm{CrN}$ films grown at different temperatures can fully be explained in terms of the different Young moduli. Furthermore, we show that these $\mathrm{CrN}$ thin films together with DLC and diamond films exhibit the same linear dependence between the nanoscopic friction coefficient and the inverse of the Young modulus. These observations show that there are materials with similar values of critical shear strength and that for such materials nanoscopic wearless friction is univocally determined by the Young modulus.

The surface topography of the films and the friction forces have been investigated by an AFM (AutoProbeTM M5), with a sliding velocity of $1 \mu \mathrm{m} / \mathrm{s}$, at room temperature and $44 \%$ of relative humidity. We used V-shaped silicon cantilevers (Ultralevers B) and silicon conical tips. The normal load and the friction force are proportional to the normal and lateral deflections of the cantilever, and they are recorded simultaneously. Zero normal load is defined as the position where the cantilever is not bent. Other experimental details can be found in Ref. 12. No evidence of tip and sample wear (seen as a change of friction or topography with test duration on the same scanned area) was observed during the course of the experiments.

DLC thin films have been grown by laser ablation and their structural properties have been characterized as described in Refs. 13 and 14. Polycrystalline $\mathrm{CrN}$ thin films have been deposited by magnetron sputtering at different temperatures $\left(T_{G}=300,530\right.$, and $\left.770 \mathrm{~K}\right) .{ }^{15}$ The temperature induces changes in the surface and bulk morphology of the films, however, leaving unchanged the electronic structure and chemical composition as determined by $\mathrm{x}$-ray photoelectron spectroscopy and spectroscopic ellipsometry. ${ }^{16}$ The average grain size decreases from about $40 \mathrm{~nm}$ for $T_{G}$ $=300 \mathrm{~K}$ to about $30 \mathrm{~nm}$ for $T_{G}=770 \mathrm{~K}$. The rms surface roughness measured on $1 \mu \mathrm{m}^{2}$ slightly decreases by increasing $T_{G}$ from about 5 to $4 \mathrm{~nm}$. The surface morphology of the 
TABLE I. Summary of the results obtained for $\mathrm{CrN}$ films.

\begin{tabular}{cccccc}
\hline \hline & & $\mu$ & $F_{\text {adh }}(\mathrm{nN})$ & $\mu$ & $F_{\text {adh }}(\mathrm{nN})$ \\
$T_{G}(\mathrm{~K})$ & $E(\mathrm{GPa})$ & $(S=1 \mu \mathrm{m})$ & $(S=1 \mu \mathrm{m})$ & $(S=25 \mathrm{~nm})$ & $(S=25 \mathrm{~nm})$ \\
\hline 300 & 170 & $0.42(2)$ & $0.6(3)$ & $0.40(4)$ & $0(1)$ \\
530 & 220 & $0.37(2)$ & $1.2(3)$ & $0.36(8)$ & $3(1)$ \\
770 & 280 & $0.22(2)$ & $7.6(3)$ & $0.22(3)$ & $7(1)$ \\
\hline \hline
\end{tabular}

samples grown at $T_{G}=770 \mathrm{~K}$ shows a higher packing density in agreement with the structure zone model. ${ }^{17}$ This is also confirmed by $\mathrm{x}$-ray reflectivity measurements: the mass density increases from $5 \mathrm{~g} / \mathrm{cm}^{3}$ for $T_{G}=300 \mathrm{~K}$ to $5.7 \mathrm{~g} / \mathrm{cm}^{3}$ for $T_{G}=770 \mathrm{~K} .{ }^{16}$ This densification process in the films is accompanied by an increase of the nanohardness and the Young modulus, ${ }^{16,18}$ as determined by nanoindentation measurements with a Berkovich-type diamond tip $^{2}$ (see Table I).

The friction force as a function of the normal load for CrN thin films grown at $T_{G}=300,530$, and $770 \mathrm{~K}$ is presented in Fig. 1(a). The data were acquired on a scan size of $S=1 \mu \mathrm{m}$ and show that the friction force increases to a very good approximation linearly with $F_{N}$ in all the investigated $\mathrm{CrN}$ films. This behavior is indicative of a multiasperity contact or of a self affine surface. ${ }^{1,19}$ In this case the relationship between friction and normal forces is given by

$$
F_{F}=\mu\left(F_{N}+F_{\mathrm{adh}}\right)
$$

where $\mu$ is the friction coefficient of the films and $F_{\text {adh }}$ is the
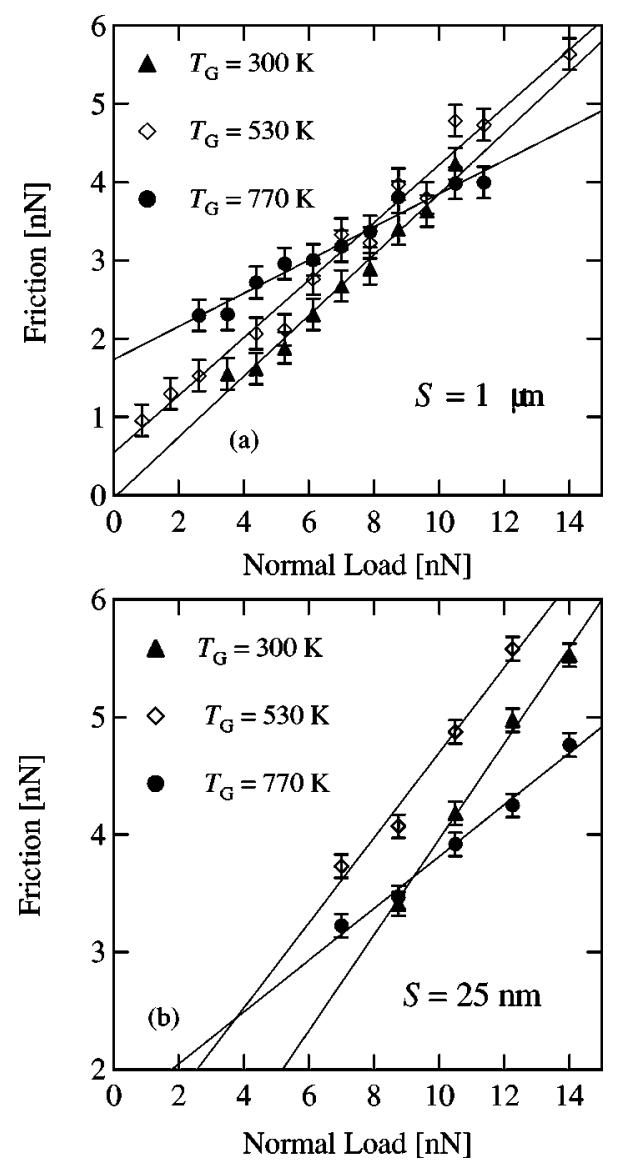

FIG. 1. Friction force as a function of $F_{N}$ for $\mathrm{CrN}$ films grown at three different $T_{G}$. The scan size is $1 \mu \mathrm{m}$ in (a) and $25 \mathrm{~nm}$ in (b). different $T_{G}$. The scan size is $1 \mu \mathrm{m}$ in (a) and $25 \mathrm{~nm}$ in (b). Fig. 2 relating $\mu$ to $1 / E$.
Downloaded 03 Sep 2003 to 128.178.50.108. Redistribution subject to AlP license or copyright, adhesion force between the AFM tip and the sample surface. Our data show that the friction coefficient decreases with increasing $T_{G}$ (see Table I).

As a possible cause for the change of $\mu$ with $T_{G}$ we rule out the chemical surface composition and electronic structure since previous studies have shown that $T_{G}$ does not alter these properties. ${ }^{16}$ The role of surface morphology on friction has been investigated by studying the friction force on a sliding distance of $25 \mathrm{~nm}$, which is smaller than the smallest grain size of the films. Again the data shown in Fig. 1(b) display a linear behavior of $F_{F}$ with $F_{N}$ and the fits yield within the error bars identical values of $\mu$ and $F_{\text {adh }}$ as the ones found for $S=1 \mu \mathrm{m}$ (see Table I). This signifies that the changes in the surface morphology do not induce the decrease of $\mu$ with increasing growth temperature. Moreover, it is reasonable to assume that also the critical shear strength between the AFM tip and the $\mathrm{CrN}$ surfaces is the same in all our polycrystalline $\mathrm{CrN}$ films since they have the same chemical and electronic structure. These arguments suggest that the friction behavior in $\mathrm{CrN}$ films has to be understood on purely mechanical grounds, namely by a relationship between the contact area and the applied load.

In the modified Hertz theory ${ }^{8}$ the contact area between two elastic and perfectly smooth solids (e.g., a sphere and a plain) in presence of adhesive forces, ${ }^{20,21}$ can be approximated by the following relationship:

$$
A=\pi\left[\frac{3 R\left(F_{N}+F_{\mathrm{adh}}\right)}{4 E^{*}}\right]^{2 / 3},
$$

where $R$ is the sphere radius and $E^{*}=\left[\left(1-\nu^{2}\right) / E+(1\right.$ $\left.\left.-\nu_{\text {tip }}^{2}\right) / E_{\text {tip }}\right]^{-1} ; E, E_{\text {tip }}, \nu$, and $\nu_{\text {tip }}$ are, respectively, the Young modulus and the Poisson ratio of the sample and the tip. From Eqs. (1) and (3), a relationship between the friction force and $E^{*}$ is obtained

$$
F_{F}=\tau \pi\left(\frac{3 R}{4 E^{*}}\right)\left(F_{N}+F_{\text {adh }}\right)^{2 / 3} .
$$

This equation is valid in the case of a single asperity contact. However, Eq. (4) can be modified for a multiasperity contact where plastic deformations are absent. Following the model of Greenwood $^{22}$ for a finite asperity height distribution, in presence of adhesion forces, we can write

$$
F_{F}=\tau\left(\frac{\pi}{2}\right)^{1 / 2}\left(\frac{3 R^{1 / 2}}{4 E^{*} \lambda^{1 / 2}}\right)\left(F_{L}+F_{\mathrm{adh}}\right),
$$

where $\lambda$ is the rms width of the height distribution on the surfaces, which is assumed to be Gaussian. From comparison of Eq. (5) with Eq. (2) we find

$$
\mu=\frac{3}{4} \tau\left(\frac{\pi R}{2 \lambda}\right)^{1 / 2}\left[\frac{\left(1-\nu^{2}\right)}{E}+\frac{\left(1-\nu_{\mathrm{tip}}^{2}\right)}{E_{\mathrm{tip}}}\right] .
$$

For the investigated $\mathrm{CrN}$ films $\lambda$ can be considered as constant due to the similar surface roughness, and idem for $\tau$ due to the identical surface chemistry. In addition, the Poisson ratio is $\sim 0.3$ in all the materials considered in this study and thus $\left(1-\nu^{2}\right) \cong 1$. Therefore Eq. (6) shows that, for a given tip, the friction coefficients obtained on the different CrN films must be linked to their different Young moduli. This is indeed to a good approximation the case as shown in 


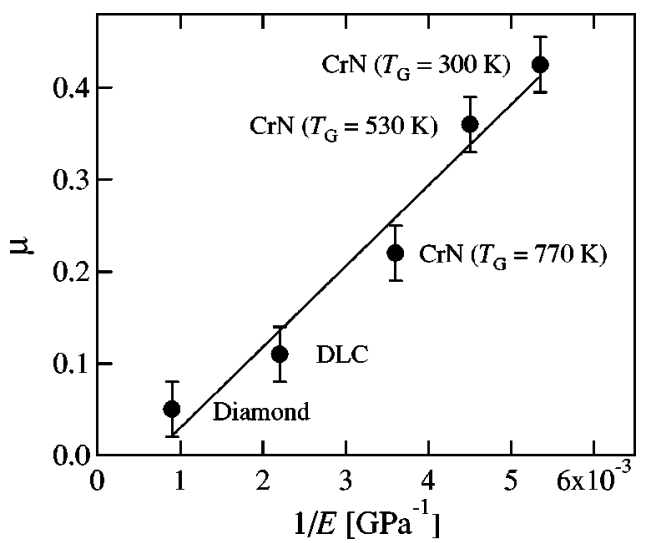

FIG. 2. $\mu$ as a function of $1 / E$ for $\mathrm{CrN}$ grown at three different temperatures, DLC, and diamond.

To draw a conclusive and more general image we have extended our study to DLC and diamond films. ${ }^{2}$ These materials are hard films, too, and they are similarly inert as our CrN films. ${ }^{16,23}$ Thus, we expect similar values for $\tau$ also for DLC and diamond films. Figure 2 shows that all the data recorded on our five samples follow a linear relationship between $\mu$ and $1 / E$. This is expected from Eq. (6) for similar values of $\tau$. The other way around, our data show that there are materials for which the friction coefficient is straightforwardly linked to the Young modulus. The fact that the linear fit shown in Fig. 2 yields $\mu \cong 0$ roughly when $1 / E \cong 0$ would imply that $E_{\text {tip }} \gg E$. This is not expected from bulk properties because the tip is silicon and $\mathrm{Si}$ Young modulus is $\approx 180 \mathrm{GPa}$. However, we note that the tip apex has a radius of curvature of about $50 \mathrm{~nm}$ and therefore under this condition the continuum contact mechanics used above in Eq. (3) to model the elastic deformation of the tip with the term (1 $\left.-\nu_{\text {tip }}^{2}\right) / E_{\text {tip }}$ can no longer be valid.

In conclusion, we have studied sliding friction of a $\mathrm{Si}$ AFM tip on $\mathrm{CrN}$ thin films grown by magnetron sputtering at different temperatures. Our study shows that the changes in the friction coefficient of these films are strictly related to the variations of their Young modulus. In particular, we have found that $\mathrm{CrN}$ films, diamond and DLC films show the same linear dependence between friction coefficient and the inverse of the Young modulus. These observations suggest that there are materials with similar values of shear strength, e.g., hard carbon based materials, for which wearless nanoscopic friction is governed in a predictable way by the Young Modulus.

The authors acknowledge F. Levy for providing them with $\mathrm{CrN}$ films and B. Persson and R. Sanjines for useful discussions.

${ }^{1}$ B. N. J. Persson, Sliding Friction: Physical Principles and Applications, NanoScience And Technology, 2nd ed. (Springer, Berlin, 200).

${ }^{2}$ B. Bhushan, Handbook of Micro/NanoTribology, 2nd ed. (CRC Press, Cleveland, 1999).

${ }^{3}$ A. Leyland and A. Matthews, Wear 246, 1 (2000).

${ }^{4}$ J. P. Gao, W. D. Luedtke, and U. Landman, Science (Washington, DC) 270, 605 (1995).

${ }^{5}$ R. M. Overney et al., Langmuir 10, 1281 (1994)

${ }^{6}$ B. Nysten, R. Legras, and J. L. Costa, J. Appl. Phys. 78, 5953 (1995).

${ }^{7}$ F. P. Bowden and D. Tabor, Friction and Lubrication of Solids: Part I (Oxford University Press, New York, 1950).

${ }^{8}$ K. L. Johnson, Contact Mechanics (Cambridge University Press, Cambridge, 1987).

${ }^{9}$ M. A. Lantz, S. J. O'Shea, M. E. Welland, and K. L. Johnson, Phys. Rev. B 55, 10776 (1997).

${ }^{10}$ R. W. Carpick, D. F. Ogletree, and M. Salmeron, Appl. Phys. Lett. 70, 1548 (1997).

${ }^{11}$ Y. L. Su and S. H. Yao, Wear 205, 112 (1997).

${ }^{12}$ E. Riedo, F. Levy, and H. Brune, Phys. Rev. Lett. 88, 185505 (2002).

${ }^{13}$ E. Riedo, F. Comin, J. Chevrier, and A. M. Bonnot, J. Appl. Phys. 88, 4365 (2000)

${ }^{14}$ E. Riedo, J. Chevrier, F. Comin, and H. Brune, Surf. Sci. 477/1, 25 (2001).

${ }^{15}$ P. Hones, R. Sanjinés, and F. Lévy, Surf. Coat. Technol. 94/5, 398 (1997).

${ }^{16}$ P. Hones, Ph.D. thesis, EPFL, Lausanne, Switzerland, 2000.

${ }^{17}$ J. A. Thornton, Annu. Rev. Mater. Sci. 7, 239 (1977).

${ }^{18}$ D. Wang and T. Oki, Thin Solid Films 185, 219 (1990).

${ }^{19}$ B. N. J. Persson, Phys. Rev. Lett. 87, 1161 (2001).

${ }^{20}$ A. Fogden and L. R. White, J. Colloid Interface Sci. 138, 414 (1990).

${ }^{21}$ U. D. Schwartz, O. Zworner, P. Koster, and R. Wiesendanger, Phys. Rev. B 56, 6987 (1997).

${ }^{22}$ J. A. Greenwood, Fundamentals of Friction (Kluwer, Dordrecht, 1992).

${ }^{23}$ Diamond surfaces are inert in air since they are passivated by hydrogen. 